\title{
Seismic Attenuation Computed from GLIMPCE Reflection Data and Comparison with Refraction Results
}

\author{
Michael P. Matheney ${ }^{1}$ and Robert L. Nowack ${ }^{2}$
}

\begin{abstract}
Instantaneous frequency matching has been used to compute differential $t^{*}$ values for seismic reflection data from the Great Lakes International Multidisciplinary Program on Crustal Evolution (GLIMPCE) experiment. The differential attenuation values were converted to apparent $Q^{-1}$ models by a fitting procedure that simultaneously solves for the interval $Q^{-1}$ values using non-negative least squares. The bootstrap method was then used to estimate the variance in the interval $Q^{-1}$ models. The shallow $Q^{-1}$ structure obtained from the seismic reflection data corresponds closely with an attenuation model derived using instantaneous frequency matching on seismic refraction data along the same transect. This suggests that the effects of wave propagation and scattering on the apparent attenuation are similar for the two data sets. The $Q^{-1}$ model from the reflection data was then compared with the structural interpretation of the reflectivity data. The highest interval $Q^{-1}$ values $(>0.01)$ were found near the surface, corresponding to the sedimentary rock sequence of the upper Keweenawan. Low $Q^{-1}$ values $(<0.0006)$ are found beneath the Midcontinent rift's central basin. In addition to structural interpretation, seismic attenuation models derived in this way can be used to correct reflection data for dispersion, frequency and amplitude effects, and allow for improved imaging of the subsurface.
\end{abstract}

Key words: Seismic attenuation, seismic $Q$, Midcontinent rift.

\section{Introduction}

Seismic attenuation causes a preferential loss of the higher frequencies, as well as phase distortions, in a seismic signal with increasing propagation distance. These effects can cause discrepancies between seismic reflection data and well-log data. Several techniques have recently been developed to incorporate attenuation in the processing of seismic reflection data. For example, inverse- $Q$ filtering (HARGREAVES and CALVERT, 1991; Bickel, 1993) and dispersion corrections (DurEN and Trantham, 1997) use an attenuation model with depth to correct pre-stack seismic data for frequency loss and phase distortion. $Q$-deconvolution (BICKEL and NATARAJAN, 1985) has been shown to effectively remove amplitude, frequency, and

\footnotetext{
${ }^{1}$ Exxon Exploration Company, Houston, TX, 77253. E-mail: matheney@geo.eas.purdue.edu

${ }^{2}$ Purdue University, Dept. of Earth and Atmospheric Science, West Lafayette, IN 47907. E-mail: nowack@geo.eas.purdue.edu
} 
dispersion effects resulting from wave propagation in an attenuating medium. Amplitude statics can be applied to correct for amplitude differences in the reflection data that are due to near-surface, localized low $Q$ areas (BRzOSTOWSKI and MCMECHAN, 1992). In addition, migration algorithms have incorporated attenuation information to provide improved images of the subsurface (SOLLIE and MitTet, 1994).

While there has been increased interest in using attenuation information for the processing of reflection data, most studies have assumed that an attenuation model is known, and relatively few studies have concentrated on the determination of $Q$ models. In this study, an instantaneous frequency matching procedure similar to that developed by MAtheney and Nowack (1995) is used to determine the differential attenuation between a reference arrival and other arrivals on a seismic reflection trace. The differential attenuation values are then used to obtain the interval $Q$ structure.

The instantaneous frequency matching procedure is first tested using synthetic reflection data. This allows for an analysis of the effects of noise and interference on the derived attenuation values. The method is then applied to seismic reflection data of the 1986 GLIMPCE Lake Superior seismic experiment and used to compute interval $Q^{-1}$ values. The resulting $Q^{-1}$ model is then compared to an independently derived attenuation model obtained from seismic refraction data by MATHENEY et al. (1997) along the same transect. Finally, the derived attenuation structure is compared with the interpreted reflectivity structure from CANNON et al. (1989).

\section{Method of Analysis}

\section{Instantaneous Frequency Matching}

In order to apply the instantaneous frequency matching procedure of MATHENEY and NowACK (1995) to seismic reflection data, a reference pulse is first selected and windowed from a reflection trace. The instantaneous frequency of the reference pulse is then matched with the instantaneous frequency of each reflection on the seismic trace. This is performed by attenuating the reference pulse using a nearly-constant $Q$ attenuator, and comparing the instananeous frequency of the attenuated reference pulse to an observed seismic pulse. The advantage of using a nearly constant $Q$ operator, as opposed to an exactly constant $Q$ operator (KJARTANSSON, 1979), is that for the nearly constant $Q$ operator a $t^{*}$ parameter can be expressed as an integration of the attenuation along the ray. The transfer function for the nearly constant $Q$ operator can then be written as

$$
A(\omega)=e^{i \omega\left[T-\left(t^{*} / \pi\right) \ln \left(\omega / \omega_{r}\right)\right]} e^{-(\omega / 2) t^{*}},
$$


where $T=\int 1 / c(s) d s$ is the travel time, $t^{*}=\int Q^{-1}(s) / c(s) d s$ is the integrated attenuation along the ray, $c(s)$ is the velocity at the reference frequency $\omega_{r}, Q^{-1}(s)$ is the seismic quality factor, and $s$ is the path length along the ray (AKI and RICHARDS, 1980). The amount of attenuation for this operator is controlled by adjusting the $t^{*}$ value. By matching the instantaneous frequency of the attenuated reference pulse at its peak envelope amplitude to the instantaneous frequency of the observed pulse, the differential attenuation between the two arrivals can be obtained.

The instantaneous frequency matching procedure assumes that the locations of the reflections on a seismic trace are known. Due to the large number of traces in a typical seismic reflection survey and the numerous reflections on each trace, it is impractical to manually pick each reflection. Since the instantaneous frequency matching procedure uses the frequency values at peaks of the envelope amplitude, it is sufficient to consider the peaks of the envelope on a seismic trace as possible locations of seismic arrivals. By applying several checks to the current time position along the trace, it can be determined if a given peak of the envelope is a well isolated prominent reflection.

In order to identify the largest, envelope amplitude arrival over a small window on the seismic trace, the current peak envelope location is checked against adjacent peaks of the envelope. The decay of the envelope before and after the current peak envelope location is then computed. If the envelope does not decrease by at least $30 \%$ of the maximum on each side of the peak, then it is assumed that the current peak location is contaminated by interfering arrivals and is not used. The interference between arrivals can cause errors in the determination of differential attenuation along a seismic trace (MATHENEY and NowACK, 1995). A final check to determine if a specific envelope peak is an isolated reflection peak is performed by windowing out the current arrival using a cosine-bell taper and computing its amplitude spectrum. The amplitude spectrum is then compared against the amplitude spectrum from a noise sample obtained prior to the first arrival on the trace. If the amplitude spectrum of the current arrival is above the noise level over a bandwidth of at least $15 \mathrm{~Hz}$, then it is considered as a possible reflection peak. Otherwise, the envelope peak is excluded. The minimum bandwidth of $15 \mathrm{~Hz}$ was empirically determined, and is dependent on the frequency content of the reflection arrivals.

For the application of the instantaneous frequency matching procedure, windowed segments of the trace are filtered based on the frequency content of the reflection arrival and the noise level of the trace. The instantaneous frequency of the reference pulse is then matched to the instantaneous frequency of the filtered, reflection arrival at its envelope peak. This process was shown to provide a more accurate estimate of attenuation than spectral ratios, particularly for data with lower signal-to-noise-ratios (MATHENEY and NowACK, 1995). The matching procedure results in differential attenuation $t^{*}$ values as a function of time along a seismic reflection trace which can then be used to obtain interval $Q^{-1}$ values. 


\section{Estimation of Interval $Q^{-1}$ Values}

In order to obtain interval $Q^{-1}$ values from seismic reflection data, the differential $t^{*}$ values, as well as travel times, between a reference pulse and reflected arrivals on a seismic trace are required. The travel times are obtained from the envelope peaks of the reference pulse and the reflected pulses. The differential attenuation value $\left(\Delta t_{n}^{*}\right)$ for a reflection arrival at time $t_{n}$ is given by

$$
\Delta t_{n}^{*}=t^{*}\left(t_{n}\right)-t_{\text {ref }}^{*}\left(t_{0}\right)=\int_{0}^{t_{n}} Q^{-1}\left(t^{\prime}\right) d t^{\prime}-\int_{0}^{t_{0}} Q^{-1}\left(t^{\prime}\right) d t^{\prime} .
$$

Assuming nearly normal incidence, this can be approximated by

$$
\Delta t_{n}^{*}=t^{*}\left(t_{n}\right)-t_{\text {ref }}^{*}\left(t_{0}\right) \cong \int_{t_{0}}^{t_{n}} Q^{-1}\left(t^{\prime}\right) d t^{\prime}=Q_{n}^{-1^{\text {avg }}}\left(t_{n}-t_{0}\right),
$$

where $Q_{n}^{-1 \text { avg }}$ is the average $Q^{-1}$ value between the reference pulse at $t_{0}$ and the $n$-th reflection pulse at $t_{n}$. The average attenuation value $Q_{n}^{- \text {avg }}$ is a time weighted average of the interval $Q^{-1}$ values for the individual layers in depth.

Since the derived $t^{*}$ values will have scatter due to interfering arrivals and noise, it is necessary to fit a curve through the $t^{*}$ data points before computing the interval $Q$ 's. For nearly zero-offset reflection data, the differential $t^{*}$ values between early and later reflected arrivals must increase with time. This can be seen from eqn. (2b) where $\Delta t_{n}^{*}$ at time $t_{n}$ will be greater than $\Delta t_{n-1}^{*}$, for $t_{n}$ greater than $t_{n-1}$ and $Q^{-1}$ greater than zero. Thus the attenuation values should increase with time down a zero offset reflection trace. In order to satisfy this constraint, as well as find a best-fitting curve through the data, a non-negative least-squares procedure (LAWSON and HANSEN, 1995) is used to fit all the observed $t^{*}$ data points simultaneously.

Figure 1a shows an example of $t^{*}$ values versus time extracted from several observed seismic reflection traces. In order to compute the best-fit curve, the $t^{*}$ data are broken into a number of constant time segments. For each time segment the $Q^{-1}$ value is assumed to be constant. The slope for each of the time segments is then solved for simultaneously giving the interval $Q^{-1}$ values. In matrix form, the linear set of equations is given by

$\underset{\text { Interval \#2 }}{\longrightarrow}\left[\begin{array}{cccc}\Delta t_{1,1} & 0 & 0 & 0 \\ \Delta t_{2,1} & 0 & 0 & 0 \\ \Delta t_{1} & \Delta t_{3,2} & 0 & 0 \\ \Delta t_{1} & \Delta t_{4,2} & 0 & 0 \\ \Delta t_{1} & \Delta t_{5,2} & 0 & 0 \\ \Delta t_{1} & \Delta t_{2} & \cdot & \Delta t_{m-1, n} \\ \Delta t_{1} & \Delta t_{2} & \cdot & \Delta t_{m, n}\end{array}\right]\left[\begin{array}{l}Q_{1}^{-1} \\ Q_{2}^{-1} \\ \cdot \\ \cdot \\ Q_{n}^{-1}\end{array}\right]=\left[\begin{array}{l}\Delta t_{1}^{*} \\ \Delta t_{2}^{*} \\ \cdot \\ \cdot \\ \cdot \\ \Delta t_{m}^{*}\end{array}\right]$,


where $\Delta t_{j}$ is the length of time of the $j$-th time interval, $\Delta t_{i, j}$ is the incremental time between the observed data point $(i)$ and the start of the current time interval $(j)$, $Q_{j}^{-1}$ is the interval $Q^{-1}$ value for the $j$-th time interval, $\Delta t_{i}^{*}$ is the $i$-th data point, $m$ is the total number of $t^{*}$ data points for all time intervals, and $n$ is the number of time intervals. This problem is then solved using non-negative least squares in which all the $Q_{j}^{-1}$ values must be non-negative. The resulting solution gives the interval $Q^{-1}$ values for all the layers simultaneously, with a constraint of positive slope and a minimization of the squared errors between the observed data and the line segements. Figure $1 \mathrm{~b}$ shows the computed best-fit curve for the data shown in Figure 1a. The slope of each of the line segments is the $Q^{-1}$ value for that layer.

\section{Bootstrap Method for Error Estimation}

An advantage of using curve fitting for the estimation of the interval $Q^{-1}$ values is that the fitting procedure allows for an estimation of the uncertainties in the $Q^{-1}$

(a)

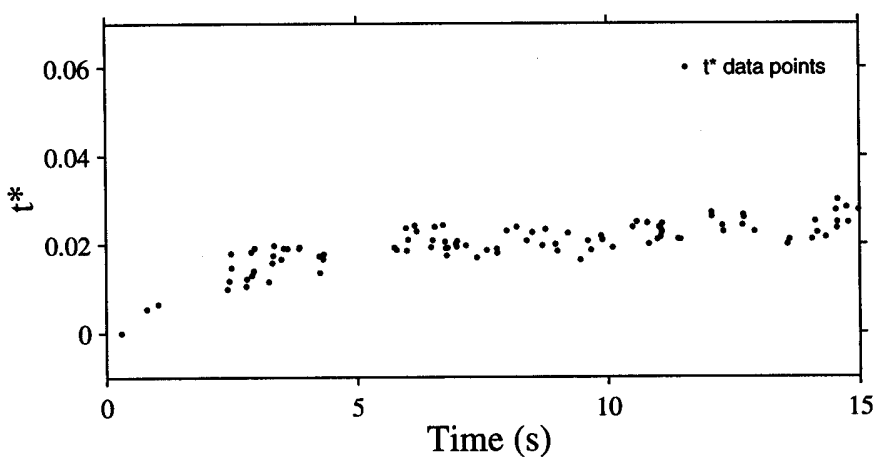

(b)

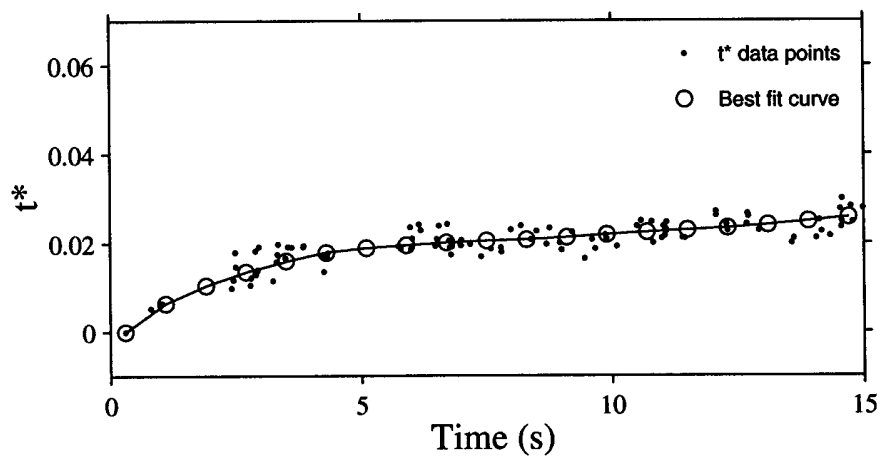

Figure 1

(a) Differential $t^{*}$ values extracted from several observed seismic traces. (b) Differential $t^{*}$ values shown in (a) with the computed best-fit curve using non-negative least squares. 
model. This can be accomplished by using the bootstrap resampling method. For the bootstrap method, the original $t^{*}$ data set is randomly resampled many times, and the model fit for each of these resampled data sets provides information about the model variance (EFRON and TIBSHIRAMI, 1993). The resampling method used for bootstrapping is the random selection of $m$ data points from the original set of $m$ data. This implies that the resampled data sets can contain a given data point more than once. The bootstrap's estimator of standard deviation $\left(\hat{\sigma}_{\text {boot }}\right)$ can be determined using a large number of realizations $(L)$ of bootstrap estimations $\left(\hat{\theta}_{i}^{*}\right)$, $i=1, L$. In our case, the bootstrap estimator $\left(\hat{\theta}_{i}^{*}\right)$ is the $Q^{-1}$ value for each layer. The bootstrap estimate of standard deviation is then

$$
\hat{\sigma}_{\mathrm{boot}}=\left[\frac{1}{L-1} \sum_{i}\left(\hat{\theta}_{i}^{*}-\bar{\theta}\right)^{T}\left(\hat{\theta}_{i}^{*}-\bar{\theta}\right)\right]^{1 / 2}
$$

where

$$
\bar{\theta}=\sum_{i} \hat{\theta}_{i}^{*} / L
$$

(TichelaAR and RUfF, 1989). For the error calculations presented below, one hundred resamplings $(L=100)$ have been used for estimating the standard deviation. This value of $L$ was found to give results similar to those obtained from larger resampling values for this data.

\section{Synthetic Data Examples}

The method for computing interval $Q^{-1}$ values described above was first tested using synthetic data examples. Figure 2 shows the velocity and attenuation models used to produce synthetic seismic data. The velocity and $Q^{-1}$ models include a thin, low velocity and high $Q$ layer at the surface corresponding to the water layer. Directly beneath this layer both the velocity and the $Q^{-1}$ values increase. At greater depths, the velocity continues to increase and the $Q^{-1}$ values decrease. Figure 3 delineates 60 synthetic seismic traces generated employing ray methods. Amplitudes have been scaled using AGC for display purposes, but true amplitudes are used for the computation of differential $t^{*}$ values. For this computed seismic section, the source-receiver offset for the first trace is 232 meters and the receiver spacing is 25 meters. The seismic section consists of the eleven primary reflections from the layered model shown in Figure 2 and eight water column multiples associated with each primary reflection. The primary effect of the $Q^{-1}$ model is to broaden the pulses. The effect of the attenuation model on the reflection coefficients has not been included for the synthetic examples since this has been shown to have a small effect except in cases of very high $Q^{-1}$ materials or very small velocity contrasts (BOURbiÉ and Nur, 1984). 
SYNTHETIC MODEL

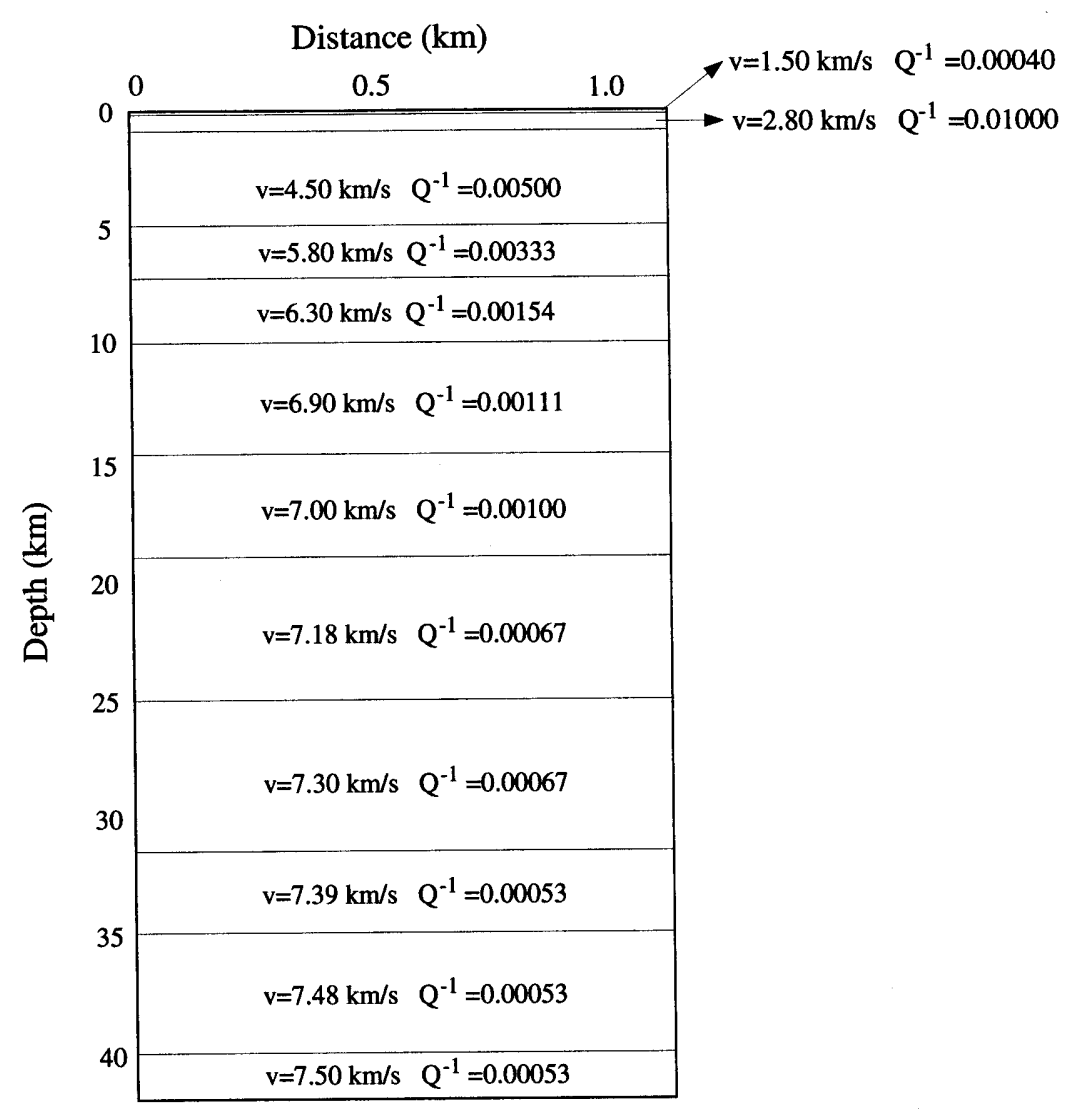

Figure 2

Velocity and attenuation models used to generate synthetic reflection seismograms.

In Figure 4, band-passed Gaussian noise is added to each trace to simulate real seismic data. A mute is applied to the first part of the traces in order to remove as much of the water reverberation as possible. The shot gather is then dip filtered using a slope cutoff of $-0.015 \mathrm{~s} /$ trace and $0.015 \mathrm{~s} /$ trace.

In order to remove the water reverberations from the primary reflections, the seismic gather is deconvolved using a gap deconvolution. For this method, the seismic trace is modeled as a sequence of primary reflections plus water reverberations at the source and receiver. For each primary reflection, this has an impulse response of

$$
g(t)=\sum_{n=0}^{\infty}(-1)^{n}(n+1) R^{n} \delta(t-n \tau)
$$




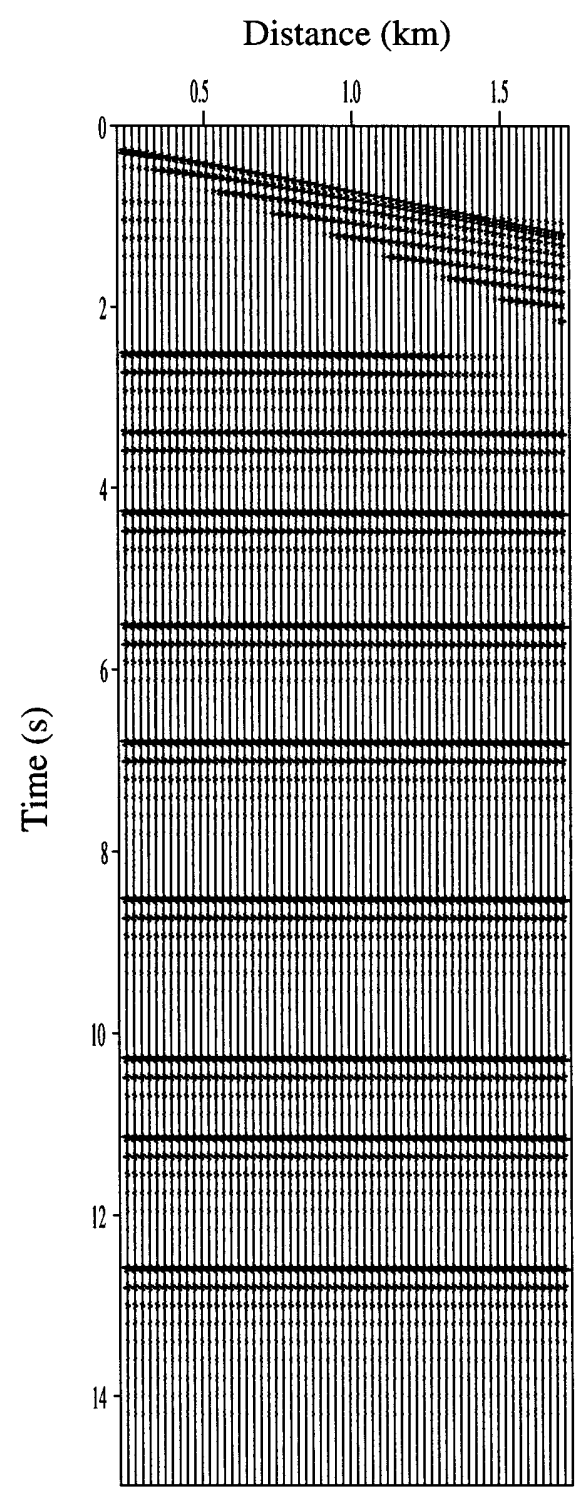

Figure 3

Synthetic seismic shot gather with eleven primary reflections and their associated water reverberations. AGC has been applied.

where $\tau$ is the two-way travel time from the water bottom to the surface and $R$ is the reflection coefficient at the water bottom. The water bottom multiple portion of the seismic record can be removed by convolving the traces with an inverse filter with an impulse response of 


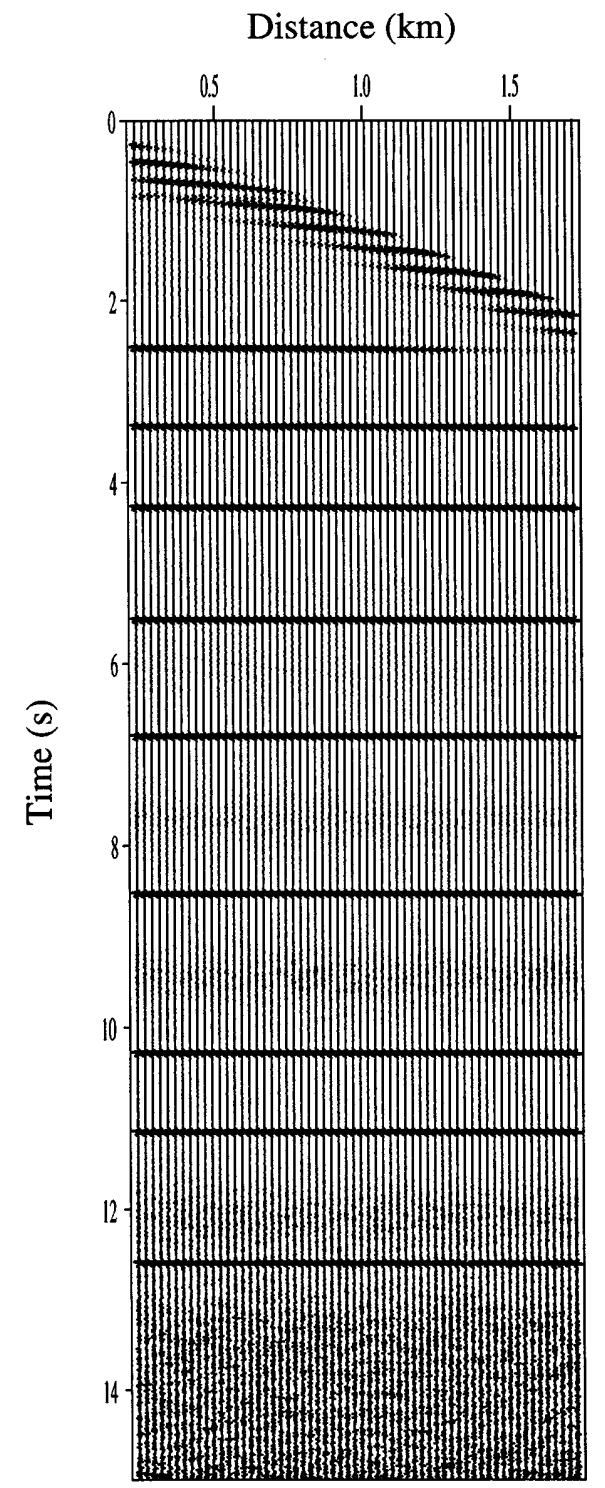

Figure 4

Noisy synthetic seismic shot gather with eleven primary reflections. Dip filtering and gap deconvolution have been applied to remove water bottom multiples. The data is plotted with an AGC of the amplitudes.

$$
h(t)=\delta(t)+2 R \delta(t-\tau)+R^{2} \delta(t-2 \tau)
$$

(BACKUS, 1959). In order to implement this deconvolution operator, the two-way travel time in the water column and the reflection coefficient of the water bottom 
must be known. In the synthetic example of Figure 4 the two-way travel time through the water column is $0.2 \mathrm{~s}$ and the water bottom reflection coefficient is 0.3 . Using these parameters to deconvolve the noisy synthetic data results in the shot gather shown in Figure 4. After deconvolution, the water reverberations for each primary reflection have been greatly reduced without affecting the pulse shape of the primary reflections.

The instantaneous frequency matching procedure was applied on traces 5 through 20 of Figure 4 to calculate the differential $t^{*}$ values. Figure 5 a shows the extracted $t^{*}$ values $(\cdot)$ along with the correct $t^{*}$ values $(+)$ and the best-fit curve $(\bigcirc)$ through the $t^{*}$ data points. For this shot gather, there is scatter in the computed $t^{*}$ values associated with the noise and processing of the gather. Also, there are several mispicks early in the seismic traces due to noise generated by the processing of the shot gather. Figure $5 \mathrm{~b}$ shows the interval $Q^{-1}$ model (solid line) and the two standard deviation uncertainties (short-dashed lines) in the model computed using the bootstrap method, along with the true $Q^{-1}$ model (long-dashed line). Although

(a)

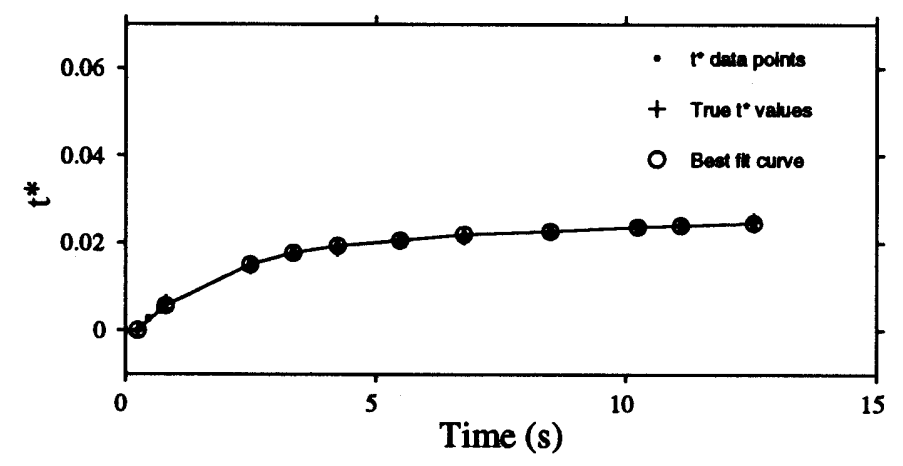

(b)

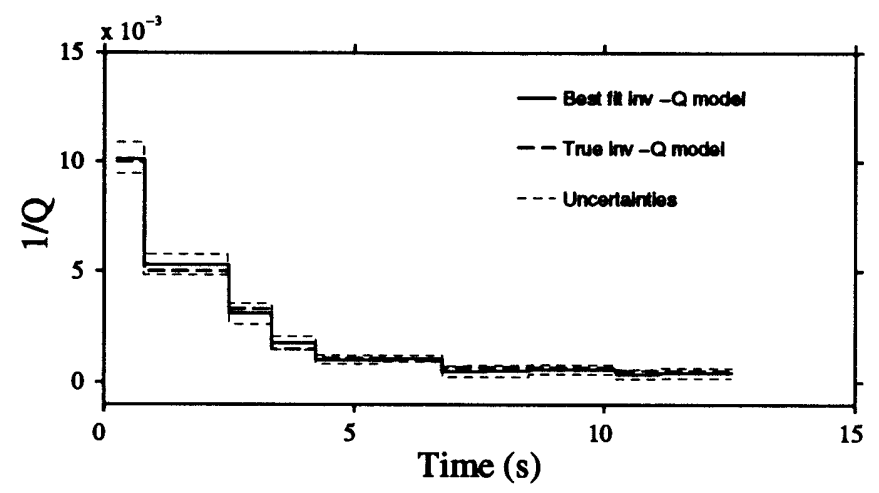

Figure 5

(a) Differential attenuation values with the best-fit curve through the $t^{*}$ data points. (b) Inverse- $Q$ model computed from the attenuation values in (a). 
(a)

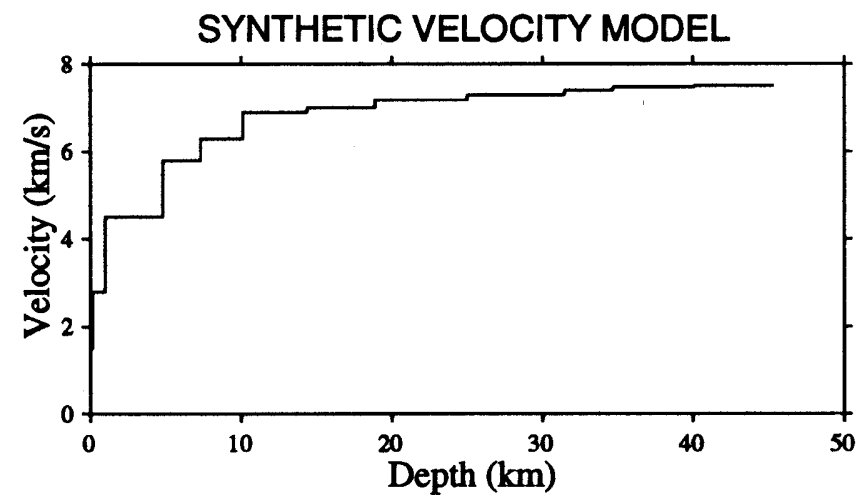

(b)

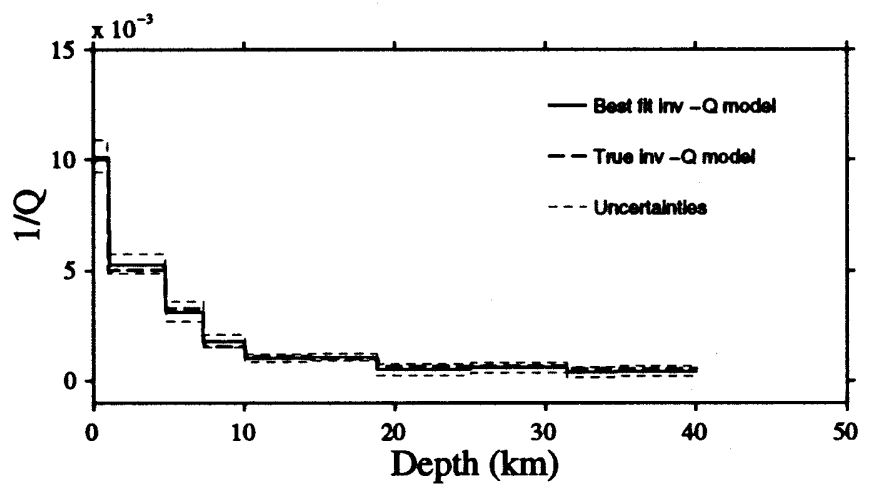

Figure 6

(a) Velocity model used to convert time to depth. (b) Inverse- $Q$ model versus depth.

the uncertainties in the model are slightly larger than in noise-free examples, the estimated and true $Q^{-1}$ models agree to within the error bounds obtained by the bootstrap method. Predictive deconvolution could have been used to remove the reverberations. However, testing with synthetic, as well as real data, demonstrated predictive deconvolution to be more likely to disrupt the frequency content of the primary reflections than the analytic deconvolution operator in eqn. (7). Any change in the frequency of the arrivals will cause erroneous differential $t^{*}$ calculations.

For a direct comparison of the extracted $Q^{-1}$ model with the original model employed computing the synthetic data (Fig. 2), the $Q^{-1}$ versus time model of Figure $5 \mathrm{~b}$ can be converted to depth using an estimated velocity model. By calculating the average velocity to different depths, the travel time to any depth can be computed. Figure 6 a displays the velocity model used in computing synthetics. In figure $6 \mathrm{~b}$ the best-fit $Q^{-1}$ model converted to depth is shown by the solid line, the short-dashed line is the uncertainty in the model, and the long-dashed line is the true $Q^{-1}$ model. 
Seismic Reflection Results

The instantaneous frequency matching procedure described above is now applied to data from the 1986 GLIMPCE Lake Superior reflection experiment. Figure 7 is a map of line A showing the shot number locations across the lake. For this experiment, a tuned airgun array was used as the source, and 120 hydrophones with an initial source-receiver offset of $232 \mathrm{~m}$ and a receiver spacing of $25 \mathrm{~m}$ were used

\section{LAKE SUPERIOR GLIMPCE REFLECTION LINE A}

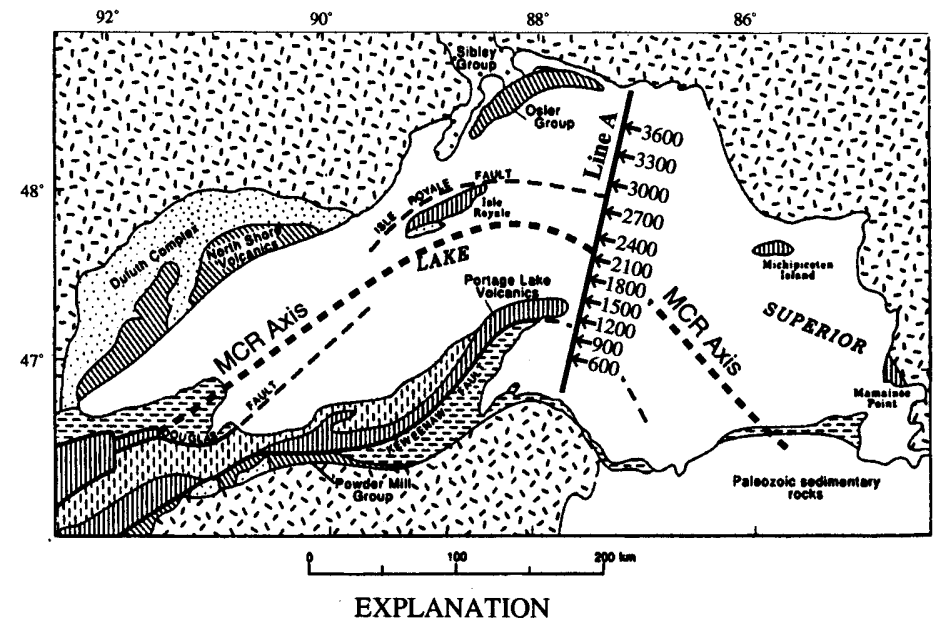

\begin{tabular}{|c|c|}
\hline$=$ & $\begin{array}{l}\text { Upper Keweenawan sedimentary rocks } \\
\text { (Bayfield Group and Jacobsville Sandstone) }\end{array}$ \\
\hline & Upper Keweenawan sedimentary rocks (Oronto Group) \\
\hline & $\begin{array}{l}\text { Middle Keweenawan } \\
\text { (Basalt flows and sedimentary rocks/Gabbroic intrusives) }\end{array}$ \\
\hline & $\begin{array}{l}\text { Lower Keweenawan } \\
\text { (Basalt flows and underlying sedimentary rocks) }\end{array}$ \\
\hline & Lower Keweenawan? (Sibley Group) \\
\hline & Archean and Lower Proterozoic crystalline rocks \\
\hline
\end{tabular}

Figure 7

Map of the Lake Superior area showing the probable lithologies and the location of line A of the GLIMPCE experiment. Shot number locations used for computing the attenuation models are specified along line A. The dashed line shows the axis of the Midcontinent rift (MCR) (Geology adapted from CANNON et al., 1989). 


\section{DATA PROCESSING FLOW CHART}

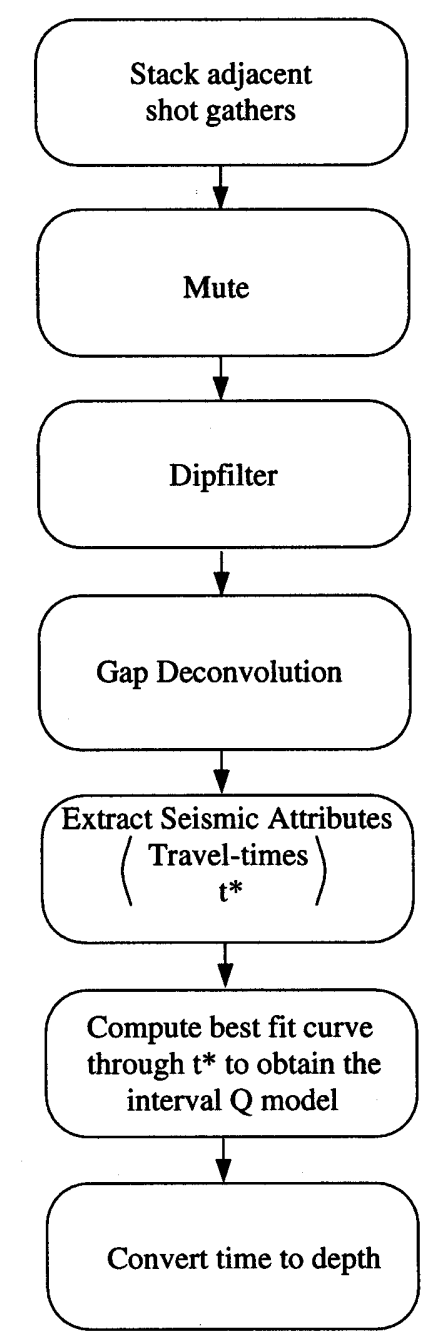

Figure 8

Processing steps used to obtain interval $Q$ values.

to record the data. For shot locations less than 2300 (see Fig. 7) a shot spacing of $50 \mathrm{~m}$ was used and for the other shots a spacing of $62.5 \mathrm{~m}$ was used. The first fifteen seconds of the seismic reflection records at eleven locations across Lake Superior are used to compute $Q^{-1}$ models. These locations are centered at shot locations 600, 900, 1200, 1500, 1800, 2100, 2400, 2700, 3000, 3300, and 3600 shown in Figure 7. 
Figure 8 shows the processing steps that were applied to the reflection data. The first step was to stack traces from adjacent shot gathers to increase the signal-tonoise ratio. Without the stacking of shot gathers, the determination of $t^{*}$ values below several seconds would have been impossible due to the low signal-to-noise ratio of the reflections. Before stacking of the shot gathers, the water depth variability was checked and shot and receiver locations with water depths that varied by more than 6 meters were not included in a given stack. Large depth variations would cause a misalignment of the reflections from adjacent shots and also make the gap deconvolution ineffective. Shot locations near 1500 and 2700 had too large a variation in the lake bathymetry and were excluded from the differential $t^{*}$ computations. At the other nine locations, 30 to 45 shot gathers were stacked depending on the lake bathymetry. This corresponds to stacking over a horizontal distance of between $1.5 \mathrm{~km}$ and $2.8 \mathrm{~km}$ for each location. After stacking, a mute was applied to the start of each shot gather. The time for the mute increased from $0.1 \mathrm{~s}$ for the first trace to $2.1 \mathrm{~s}$ for trace 60 . Each shot gather was then dipfiltered using a filter cutoff of $-0.015 \mathrm{~s} /$ trace and $0.015 \mathrm{~s} /$ trace.

After dipfiltering, the shot gathers were gap deconvolved using the deterministic deconvolution described above. The time for the gap deconvolution operator was estimated from the original experiment logs which gave the water depths. This was checked against the water bottom reflection times for each shot gather. The reflection coefficient for the deconvolution operator was estimated using a velocity of $1450 \mathrm{~m} / \mathrm{s}$ (PRESS, 1966) for the velocity of water with a density of $1000 \mathrm{~kg} / \mathrm{m}^{3}$. The velocity of the subsurface bottom was estimated from the seismic refraction results of MATHENEY and NowACK (1997). The density of the lake bottom was obtained from the density-velocity relationship of GARDNER et al. (1974). Figure 9 shows shot gather location 3600, which has been fully processed using deconvolution parameters of $0.198 \mathrm{~s}$ for the time in the deconvolution operator, and a reflection coefficient of 0.45 for the subsurface bottom.

Although deconvolution was used to remove some of the multiple reverberations from the water column and improve estimates of the differential $t^{*}$ values, application of the instantaneous frequency matching procedure to the undeconvolved data was found to produce similar results for the attenuation estimates. This is due to the fact that the matching algorithm uses arrivals with the maximum envelope amplitude over each small window and this eliminates some of the multiple arrivals. Also, the water column has considerably lower attenuation.

After processing the shot gathers, the instantaneous frequency matching procedure was applied to trace 5 through 20 for each of the nine shot locations. As an example, Figure 10 shows windowed arrivals from shot location 3600 in Figure 9. In this figure, the arrival at $0.3 \mathrm{~s}$ shown in (a) is extracted from the first trace and used as the reference pulse. This arrival corresponds to the water bottom reflection. The computed instantaneous frequency at the envelope peak is shown for each arrival and illustrates the general decrease in the reflected arrival's frequency with 
increasing time. This decrease in frequency is utilized by the matching procedure to obtain the differential $t^{*}$ values.

Figure 11 shows the computed differential $t^{*}$ values for the nine shot locations across Lake Superior. The computed best-fit curves are shown by the circles. A time interval of $0.8 \mathrm{~s}$ was used for the curve-fitting procedure for all the shot locations.

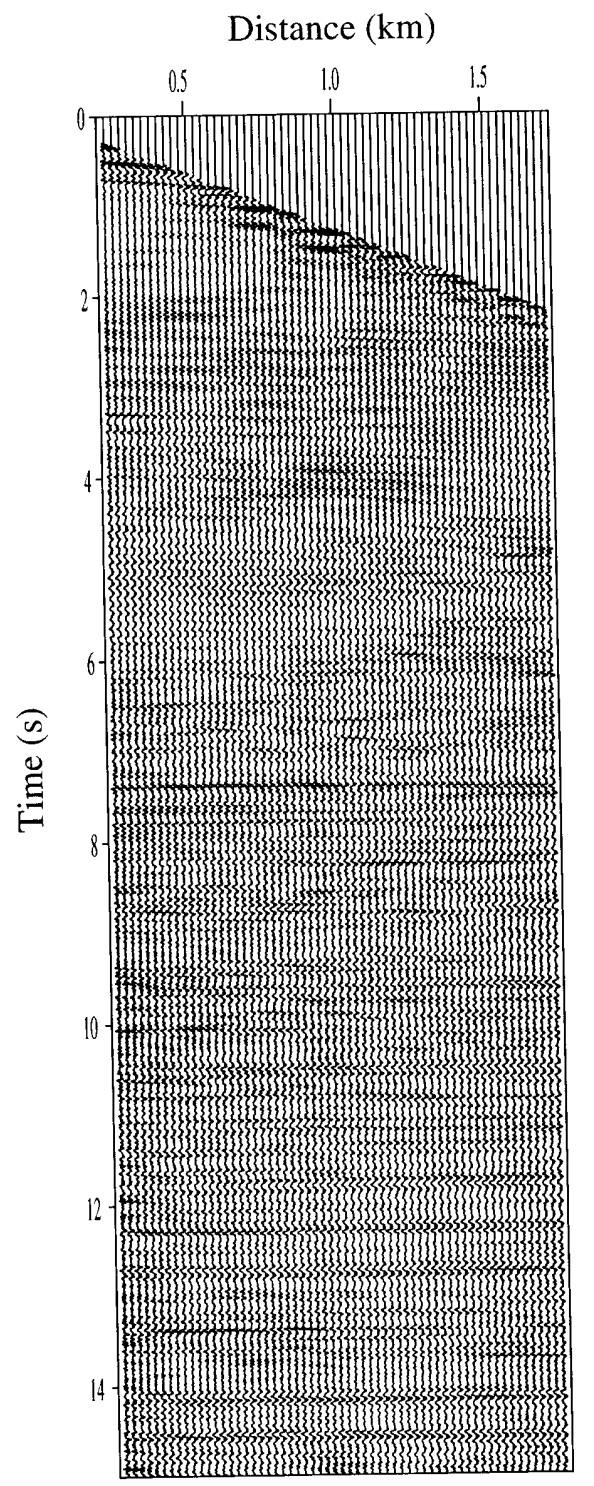

Figure 9

Processed shot gather centered around shot location 3600. The gather has been muted, dipfiltered and gap deconvolved. An AGC has been applied for display purposes. 
(a)
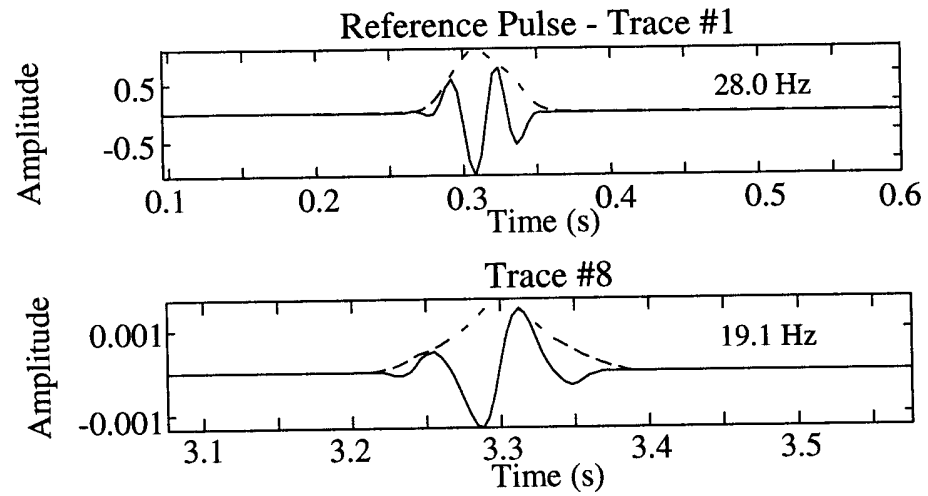

(b)

Trace \#8

(c)

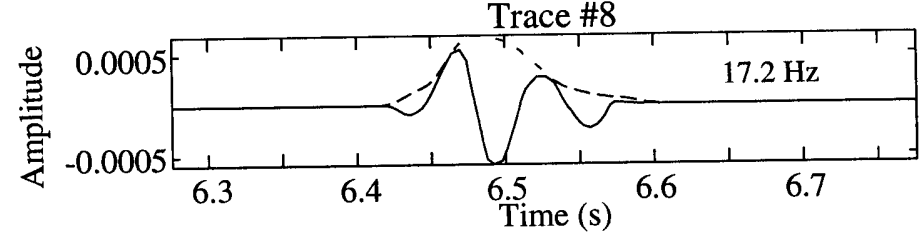

(d)

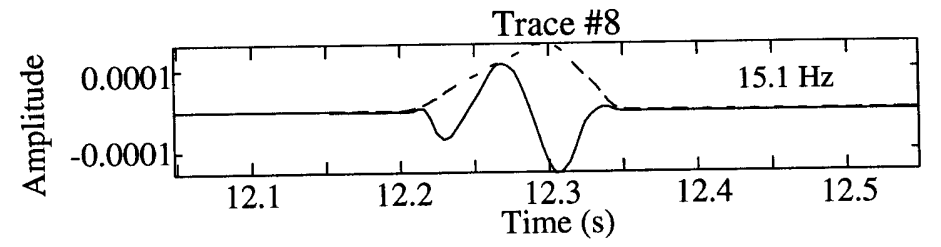

(e)

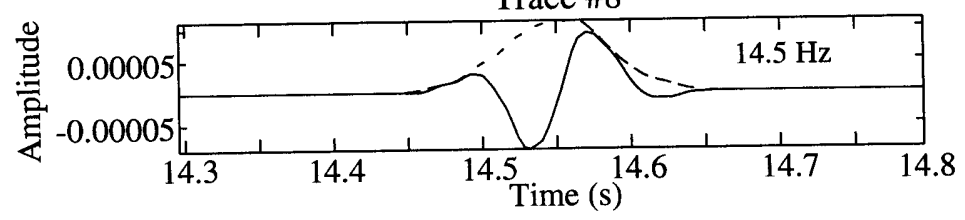

Figure 10

Windowed arrivals from the processed gather at shot location 3600 for trace number 8 . The computed envelope amplitudes are also shown along with the instantaneous frequency at the peak of the envelope.

Figure 11 shows a general decrease in the slopes of all the best-fit curves with increasing time. This is similar to that shown in the synthetic example, and results from a decrease in $Q^{-1}$ values with depth. The interval $Q^{-1}$ models determined by the best-fit curve slopes are also shown in Figure 11 along with the uncertainties in the models computed using the bootstrap method. 

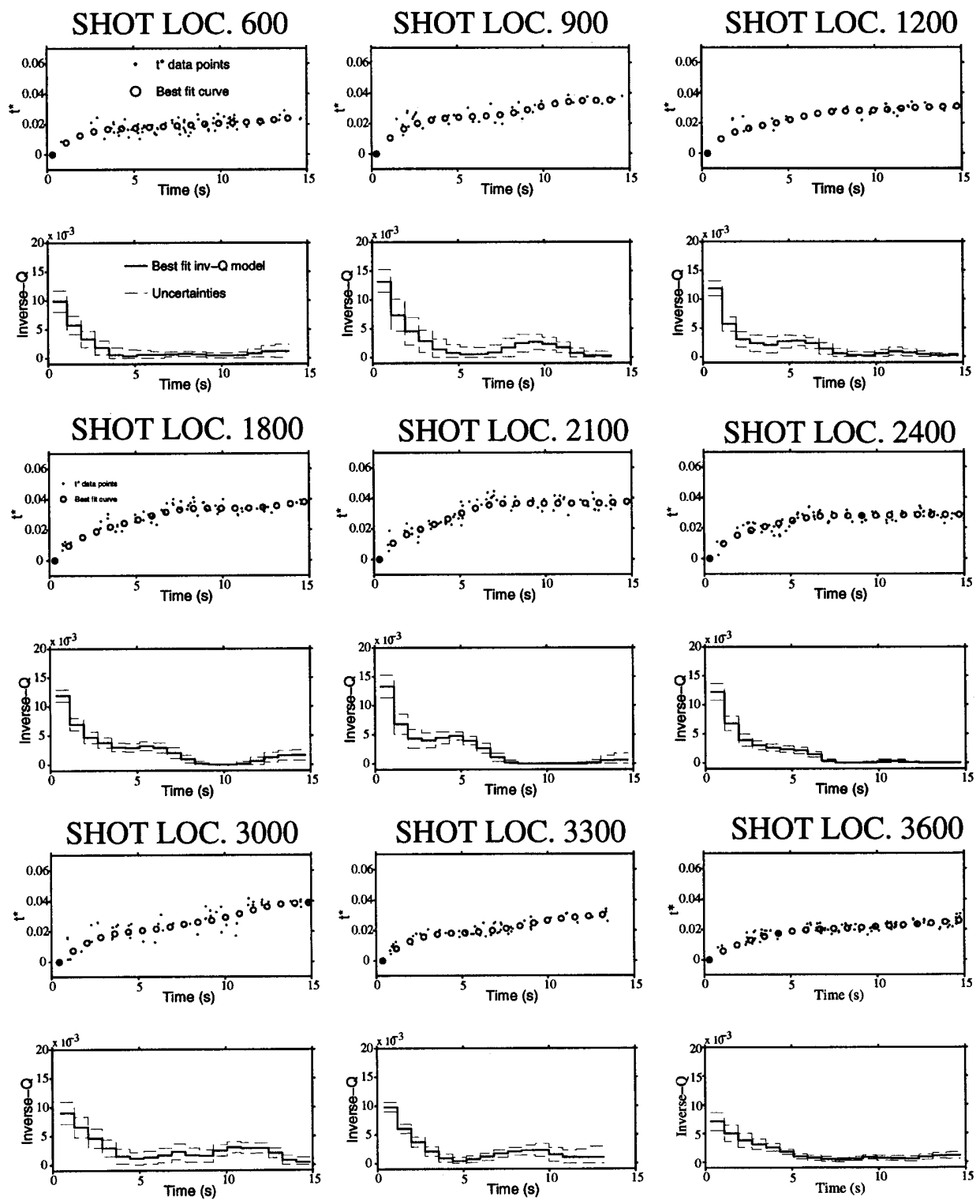

Figure 11

Differential $t^{*}$ values with the best-fit curve from the nine profiles and the resulting inverse- $Q$ models with computed uncertainties. For each shot location, the $t^{*}$ values with travel time are shown above and the estimated inverse- $Q$ with time are shown below. 


\section{Comparison with Refraction Results}

Seismic refraction data as well as reflection data were recorded during the 1986 GLIMPCE Lake Superior experiment. The refraction data along Line A were used in previous studies to determine the velocity structure (TRÉHU et al., 1991; Hamilton and Mereu, 1993; Lutter et al., 1993). In addition, MATHENEY et al. (1997) simultaneously inverted for velocity and attenuation along line A, using the method presented by NOWACK and MATHENEY (1997a) for the inversion of multiple seismic attributes.

The coincident refraction and reflection profiles provide an opportunity for the comparison of the attenuation models obtained from two independent data sets. Since the mode of wave propagation is different for refracted and reflection data, the apparent $Q^{-1}$ models derived form the two data sets may not necessarily be equivalent. SCHOENBERGER and LEVIN (1974) demonstrated that thin layering in a rock sequence can cause an increase in the apparent attenuation of reflection data due to interference of arrivals and multiples. Also refractions from interfaces with strong velocity contrasts can effect the apparent attenuation (NowACK and MATHENEY, 1997b).

In order to compare the reflection results of this study to the refraction results, the $Q^{-1}$ versus time models from the reflection data must be converted to depth. This is accomplished in a similar fashion as in Figure 6 for the synthetic data. A velocity model from the refraction studies has been used at each location to compute an average velocity and the two-way travel time to any depth is then obtained. The inverted, velocity model of MATHENEY et al. (1997) was used to convert the reflection data to depth for each of the nine $Q^{-1}$ profiles.

Figure 12 shows a comparison between $Q^{-1}$ profiles obtained from the reflection profiles for the upper $10 \mathrm{~km}$ of this study and the $Q^{-1}$ model from the refraction study of MATHENEY et al. (1997). The solid lines are $Q^{-1}$ profiles from the reflection data and the long-dashed curves are the refraction results. Uncertainties in the two $Q^{-1}$ models are also shown. For the reflection model, the average $Q^{-1}$ is computed for $0.8 \mathrm{~s}$ long time windows which results in $Q^{-1}$ layers which are approximately $2-\mathrm{km}$-thick. The refraction model is a spline interpolated model which is continuous and not layered. Despite this difference, there is good agreement between the attenuation models derived from two data sets. This implies that the wave propagation and scattering effects on apparent attenuation are similar between the two data sets.

\section{Discussion}

The GLIMPCE Lake Superior seismic experiment was a survey designed to provide insight into the Midcontinent rift (MCR), a $\approx 1.1 \mathrm{Ga}$ old paleorift which extends from Kansas up through Lake Superior and into Michigan. Line A of the Lake Superior seismic experiment transverses the MCR (see Fig. 7) and includes 


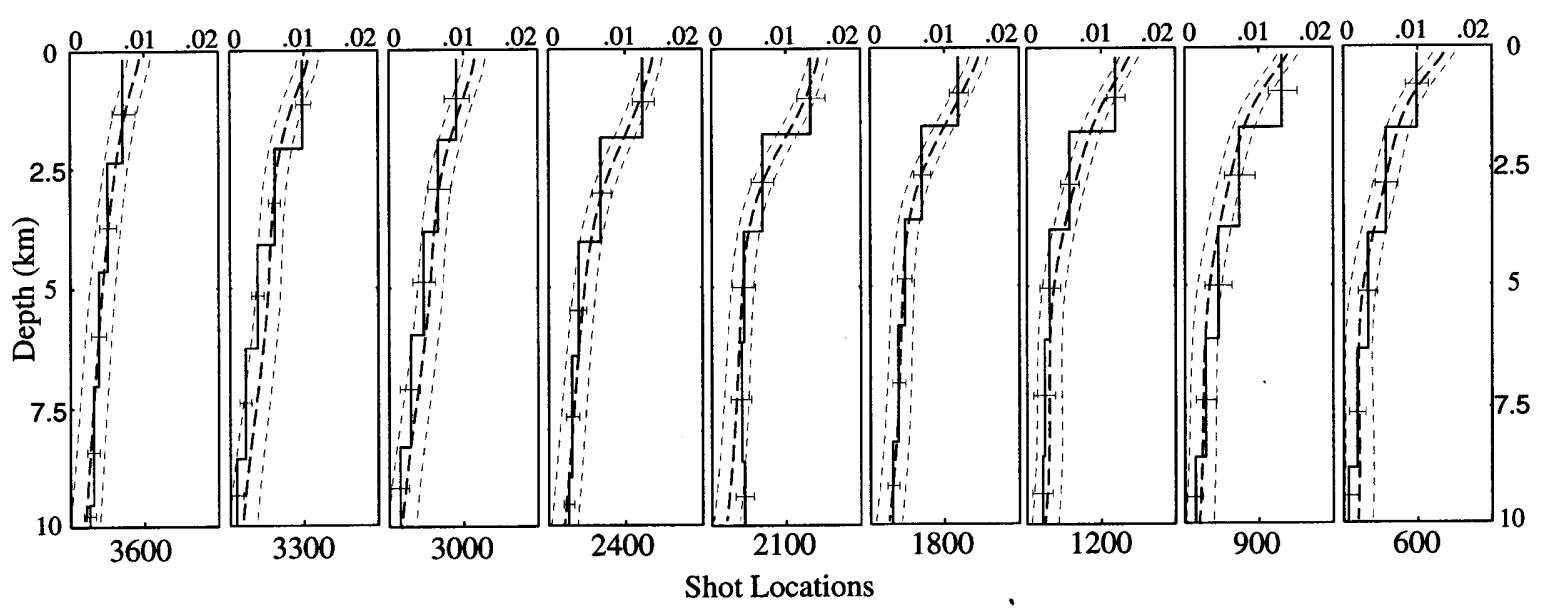

se- $Q$ model from MAtheney et al. (1997) for the nine shot locations shown in Figure 7 
coincident refraction and reflection profiles. The seismic refraction and reflection data from this line have been used to invert for the velocity and reflectivity structure of the subsurface (MATHENEY et al., 1997; LUTTER et al., 1993; SHAY and Tréhu, 1993; Hamilton and Mereu, 1993; Cannon et al., 1989). These prior results allow the attenuation model presented in this study to be related to the lithology and geologic features across the MCR.

The major shallow $(<10 \mathrm{~km})$ geologic features across the MCR include a large central rift basin between shot locations 1000 to 2600, a smaller northern rift basin extending from shot locations 2900 to 3600 , the Isle Royale fault near shot location 2850, and the Keweenaw fault near shot location 1000 (see Fig. 7). Figure 12 shows the shallow $Q^{-1}$ structure from this study. In the central basin the $Q^{-1}$ values for the first layer corresponding to depths of approximately $2 \mathrm{~km}$ are greater than 0.01 $(Q<100)$. For the deeper layers between 5 and $10 \mathrm{~km}$ in the central basin, the $Q^{-1}$ values are between 0.002 and 0.004 . Outside this basin and in the northern basin, the $Q^{-1}$ values for the first layer are less than $0.01(Q>100)$ and for the deeper layers $Q^{-1}$ values are on average lower than in the central basin. This is similar to attenuation results obtained from the refraction data by MATHENEY et al. (1997).

For the deeper regions beneath Lake Superior, the $Q^{-1}$ results shown in Figure 11 have been shaded and compared with the analysis of the reflectivity structure by CANNON et al. (1989) in Figure 13. A logarithmic gray-shade scale is used to plot the model in order to emphasize the deeper portions of the model. Overlain on the

\section{INVERSE-Q MODEL}

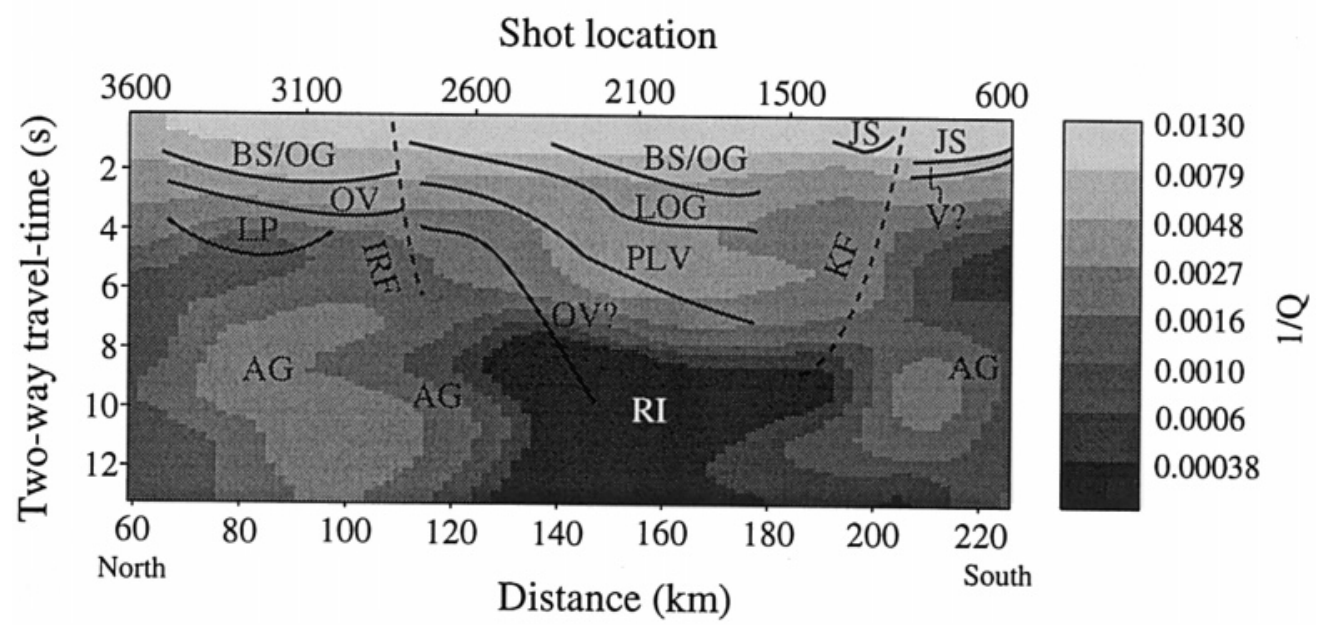

Figure 13

Gray-shade plot of the inverse- $Q$ profiles derived from the seismic reflection data. The solid and dashed lines are adapted from the interpretation of the seismic reflection data from CANNON et al. (1989). See text for an explanation of the abbreviations. Note that the vertical scale is time whereas Figure 12 is depth. 
gray-shade map is a line drawing adaptation of the interpretation of CANNON et al. (1989) along with the possible lithologies. The interpretation of CANNON et al. (1989) was derived from the reflectivity structure on the stacked and processed seismic reflection profile. The major structural features described previously, including the Isle Royale and Keweenaw faults, the large central basin and a smaller northern basin, can be seen on the reflection model of CANNON et al. (1989), as well as on the attenuation structure obtained here. The abbreviations for the different formations on Figure 13 are BG for Bayfield Group, OG for Oronto Group, OV for Osler volcanics, LOG for Lower Oronto Group, PLV for Portage Lake Volcanics, LP for Lower Proterozoic, RI for rift related rocks, JS for Jacobsville Sandstone, V for volcanics, and AG for Archean crystalline rocks.

Over the first several seconds of the model there is a layer of high $Q^{-1}$. This higher $Q^{-1}$ portion of the model corresponds with the upper Keweenawan sedimentary rocks of the Bayfield, Oronto and Jacobsville sandstone. The thickest sequence of the Oronto and Bayfield rocks occurs in the central basin around shotpoint 1600. Beneath these upper sedimentary rocks, the Lower Oronto and Portage Lake Volcanics are present in the central basin, which are primarily basaltic volcanic flows with interflow clastic sedimentary rocks. This sequence of units in the central basin is evident in the attenuation model with generally higher $Q^{-1}$ values than the adjacent Osler and lower Proterozoic volcanics outside the basin. The rocks beneath the central rift basin, noted by RI, have distinctly lower $Q^{-1}$ values and lower attenuation than the rest of the model. Previously obtained velocity models by Hamilton and Mereu (1993) and Shay and Tréhu (1993) also have significantly higher velocities beneath the central rift basin. The surrounding Archean crystalline rocks noted by AG in Figure 13 have $Q^{-1}$ values similar to those of the Osler volcanics which have interbed sedimentary rocks. In the velocity model of TRÉHU et al. (1991), there are correspondingly lower velocities at these depths near shot locations 800 and 3600 for the crystalline rocks. While lithologies seem to be a significant factor in the apparent $Q^{-1}$ values of Figure 13, scattering, interference, and thin bed layering may also have effects on apparent attenuation.

\section{Conclusion}

The method of instantaneous frequency matching has been used to compute differential $t^{*}$ values from the GLIMPCE Lake Superior reflection data. The attenuation values were converted to apparent $Q^{-1}$ models by a fitting procedure that simultaneously solved for all the interval $Q$ 's using nonnegative least squares. The bootstrap method was then used to estimate the variance in the interval $Q^{-1}$ models. The $Q^{-1}$ model obtained from the reflection data corresponds closely with a prior attenuation model derived using seismic refraction data along the same profile. This suggests that the effects of wave propagation and scattering on the apparent $Q$ models are similar for the two data sets. The $Q^{-1}$ model from the 
reflection data was then compared with the interpretation of the seismic reflectivity structure and was found to be in good agreement. Higher interval $Q^{-1}$ values ( $>0.01$ ) were found near the surface corresponding to the sedimentary rock sequences of the upper Keweenawan. Low $Q^{-1}$ values $(<0.0006)$ were found beneath the central rift basin. The surrounding crystalline formations were found to have $Q^{-1}$ values typical of those found in the basaltic flows with interflow sedimentary rocks. Finally, attenuation models derived in this way can be used to correct reflection data for dispersion, frequency and amplitude effects and allow for improved imaging of the subsurface.

\section{Acknowledgments}

The authors would like to thank Dr. Myung Lee of the United States Geological Survey for providing the seismic reflection data from the GLIMPCE experiment. Partial support was provided by National Science Foundation Grant No. EAR-9614772.

\section{REFERENCES}

Aki, K., and Richards, P., Quantitative Seismology, Theory and Methods (W. H. Freeman, New York 1989).

Backus, M. M. (1959), Water Reverberations - Their Nature and Elimination, Geophys. 24, 233-261.

Bickel, S. H. (1993), Similarity and the Inverse $Q$ Filter: The Pareto-Levy Stretch, Geophys. 58, $1629-1633$.

Bickel, S. H., and Natarajan, R. R. (1985), Plane-wave Q Deconvolution, Geophys. 50, 1426-1439.

Bourbié, T., and Nur, A. (1984), Effects of Attenuation on Reflections: Experimental Test, J. Geophys. Res. 89, 6197-6202.

Brzostowski, M. A., and McMechan, G. A. (1992), 3-D Tomographic Imaging of Near-surface Seismic Velocity and Attenuation, Geophys. 57, 396-403.

Cannon, W. F., Green, A. G., Hutchinson, D. R., Lee, M., Milkereit, B., Behrendt, J. C., Halls, H. C., Green, J. C., Dickas, A. B., Morey, G. B., Sutcliffe, R., and Spencer, C. (1989), The North American Midcontinent Rift Beneath Lake Superior from GLIMPCE Seismic Reflection Profiling, Tectonics 8, 305-332.

Duren, R. E., and Trantham, E. C. (1997), Sensitivity of the Dispersion Correction to $Q$ Error, Geophys. 62, 288-290.

Efron, B., and Tibshirami, R. J., An Introduction to the Bootstrap (Chapman and Hall, New York 1993).

Gardner, G. H. F., Gardner, L. W., and Gregory, A. R. (1974), Formation Velocity and Density: The Diagnostic Basis for Stratigraphic Traps, Geophys. 39, 770-780.

Hamilton, D. A., and Mereu, R. F. (1993), 2-D Tomographic Imaging across the North American Midcontinent Rift System, Geophys. J. Int. 112, 344-358.

Hargreaves, N. D., and Calvert, A. J. (1991), Inverse Q Filtering by Fourier Transform, Geophys. $56,519-527$.

KJartansson, E. (1976), Constant Q-wave Propagation and Attenuation, J. Geophys. Res. 84, 47374748.

Lawson, C. L., and Hansen, R. J., Solving Least-squares Problems (SIAM Press, Philadelphia 1995). 
Lutter, W. J., Tréhu, A. M., and Nowack, R. L. (1993), Application of 2-D Travel-time Inversion of Seismic Refraction Data to the Mid-continent Rift beneath Lake Superior, Geophys. Res. Lett. 20, 615-618.

Matheney, M. P., and Nowack, R. L. (1995), Seismic Attenuation Values Obtained from Instantaneous Frequency Matching and Spectral Ratios, Geophys. J. Int. 123, 1-15.

Matheney, M. P., Nowack, R. L., and TréHu, A. M. (1997), Seismic Attribute Inversion for Velocity and Attenuation Structure Using Data from the GLIMPCE Lake Superior Experiment, J. Geophys. Res. 102, 9949-9960.

Nowack, R. L., and Matheney, M. P. (1997a), Inversion of Seismic Attributes for Velocity and Attenuation Structure, Geophys. J. Int. 128, 689-700.

Nowack, R. L., and Matheney, M. P. (1997b), Extraction of Seismic Attributes from Wide-angle Synthetic Data Derived from Models with Interfaces, Trans. Am. Geophys. Un. (EOS) 78.

Press, F. In Handbook of Physical Constants (Clark, S. P., ed.) (Geological Society of America, Boulder, CO 1966).

Schoenberger, M., and Levin, F. K. (1974), Apparent Attenuation due to Intrabed Multiples, Geophys. 39, 278-291.

Shay, J., and Tréhu, A. M. (1993), Crustal Structure of the Central Graben of the Midcontinent Rift beneath Lake Superior, Tectonophysics 225, 301-335.

Sollie, R., and Mittet, R. (1994), Prestack depth migration; sensitivity to the macro absorption model, SEG Annu. Meet. Expanded Tech. Program Abstr. 64, 1422-1425.

TichelaAr, B. W., and Ruff, L. J. (1989), How Good are our Best Models? Jacknifing, Bootstrapping and Earthquake Depth, Trans. Am. Geophys. Un. (EOS) 70, 593.

Tréhu, A., Morel-Á-l'Huissier, P., Meyer, R., Hajnal, A., Karl, J., Mereu, R., Sexton, J., Shay, J., Chan, W. K., Epili, D., Jefferson, T., Shin, X. R., Wendling, S., Milkereit, B., Green, A., and Hutchinson, D. (1991), Imaging the Midcontinent Rift Beneath Lake Superior Using Large Aperture Seismic Data, Geophys. Res. Lett 18, 625-628.

(Received November 10, 1997, revised March 20, 1998, accepted April 23, 1998) 